\title{
Psychiatric epidemiology
}

\author{
Departamento de Epidemiologia \\ Faculdade de Saúde Pública da USP
}

The changes that happened in the epidemiological and psychiatric fields of work propitiated the gathering of data that were ungatherable before. The prevalence of mental and behavioral disorders, as well as the health care to patients with these pathologies are being under surveillance in several areas of the world mainly in the Unites States of America, A preliminary phase of the psyquiatric epidemiologic studies consist on in-hospital morbidity statistics. Another phase consisted on limited populational studies, confined to certain aspects of the psyquiatric disorders, such as that of alcohol and drug dependence. Finally, with the advance of diagnostic methods that could replace the psychiatric interview, with a crescent involvement of lay personnel, it was possible to obtain prevalence rates in the general population or at least in Epidemiologic Catchment Areas (ECAs). An important ex- ample of this kind of interview in the Composite International Diagostic Interview (CIDI - OMS / National Institute of Mental Health / United States), a set of questions on several topics of mental disorders, with a software that makes the diagnosis according to the International Classification of Diseases 10th Revision, and to the DSM-III-R. Another important evolution of the Psychiatric Epidemiology is the design and implementation of structured psychiatric interviews, such as that of the Schedules for Assessment in Neuropsychiatry (SCAN), a gold standard for surveys (a subsample is designed to be evaluated, and positive predictive values can be cauculated).

New horizons for research in Psychiatric Epidemiology in Brazil are discussed, mainly after the implementation of the Epidemiologic Catchment of São Paulo in 1994. 